\title{
Value Function and Optimal Rule on the Optimal Stopping Problem for Continuous-Time Markov Processes
}

\author{
Lu Ye \\ College of Economics and Management and Zhejiang Provincial Research, Center for Ecological Civilization, \\ Zhejiang Sci-Tech University, Hangzhou 310018, China
}

Correspondence should be addressed to Lu Ye; zjwzajyl@hotmail.com

Received 30 May 2017; Accepted 5 September 2017; Published 9 October 2017

Academic Editor: Antonio Di Crescenzo

Copyright (C) $2017 \mathrm{Lu}$ Ye. This is an open access article distributed under the Creative Commons Attribution License, which permits unrestricted use, distribution, and reproduction in any medium, provided the original work is properly cited.

\begin{abstract}
This paper considers the optimal stopping problem for continuous-time Markov processes. We describe the methodology and solve the optimal stopping problem for a broad class of reward functions. Moreover, we illustrate the outcomes by some typical Markov processes including diffusion and Lévy processes with jumps. For each of the processes, the explicit formula for value function and optimal stopping time is derived. Furthermore, we relate the derived optimal rules to some other optimal problems.
\end{abstract}

\section{Introduction}

Let $(\Omega, \mathscr{F}, P)$ be a complete probability space; the problem studied in this paper is to find the optimum

$$
v(x)=\sup _{\tau} \mathrm{E}^{x}\left[\mathrm{e}^{-r(\tau-t)} f\left(X_{\tau}\right) \mid \mathscr{F}_{t}\right],
$$

where $r>0$ is the discount rate and $\tau \geq t$ is a stopping time. The process $X_{t}$ is a continuous-time Markov process with starting state $X_{t}=x$. When $X_{t}$ denotes the stock price and $f(\cdot)$ is the payoff function, $v(x)$ is the pricing expression for American option (e.g., see Wong [1]). When $f(\cdot)$ is an investor's utility function about the stock price $X_{t}$, the optimum solution indicates the best time to buy or sell the stock (e.g., see McDonald and Siegel [2]).

Because of the Markov property, the value function $v(x)$ can be written as

$$
\begin{aligned}
v(x) & =\sup _{\tau} \mathrm{E}^{x}\left[\mathrm{e}^{-r(\tau-t)} f\left(X_{\tau}\right) \mid X_{t}=x\right] \\
& \triangleq \sup _{\tau} \mathrm{E}^{x}\left[\mathrm{e}^{-r(\tau-t)} f\left(X_{\tau}\right)\right],
\end{aligned}
$$

where $\mathrm{E}^{x}(\cdot)$ denotes the conditional expectation $\mathrm{E}^{x}\left(\cdot \mid X_{t}=\right.$ $x)$. The function $f(\cdot)$ is called the reward function. The possibility that $\tau=\infty$ is allowed, and making the convention that

$$
\mathrm{e}^{-r \tau} f\left(X_{\tau}\right)=0 \quad \text { if } \tau=\infty \text {, }
$$

it means that if an option is never exercised, then its reward payment is valueless for the investor.

In the paper, we are interested in determining both an optimal stopping time $\tau^{*}$ and value function $v(x)$ for a large class of reward functions. We show the conditions for reward functions and deduce the explicit optimal rules for general continuous-time Markov processes including diffusion and Lévy processes with jumps.

The study of optimal stopping time for stochastic processes, especially geometric Brownian motion, has a long history in finance literature. Under the assumption that $X_{t}$ is geometric Brownian motion, the seminal paper by McDonald and Siegel [2] puts forward the problem with the reward function $f(x)=1-x$ as a model to illustrate the financial decision making. Hu and Øksendal [3] solved the problem in multidimensional cases, when $f(x)=\sum_{i=1}^{n} x_{i}-\sum_{i=n+1}^{m} x_{i}$, but they restricted the stopping time $\tau$ in a bounded interval. Recently, Nishide and Rogers [4] extended the problem by relaxing the restriction on the stopping time. For the other forms of value functions, Pedersen and Peskir [5] solved the problem by taking some special diffusion processes.

The purpose of our work is threefold. Firstly, we intend to make clear the assumptions on reward function $f(x)$, in such a way that the explicit value can be generalized to a larger class of issues. For this purpose, throughout this article we assume that the function $f(x)$ is nonincreasing, concave and twice continuous differentiable. These properties are very 
powerful in the following proof, as we will see. Secondly, we pay attention to general Markov processes including diffusion and Lévy processes with jumps. With the help of the infinitesimal generator, we obtain an explicit formula for the value function and the stopping time. Thirdly, we find that the optimal problem (2) is equivalent to other optimal problems like

$$
\begin{aligned}
& w(x)=\sup _{\tau} \mathrm{E}^{x}\left[\int_{t}^{\tau} \mathrm{e}^{-r(s-t)} f\left(X_{s}\right) \mathrm{d} s\right], \\
& \widehat{w}(x)=\inf _{\tau} \mathrm{E}^{x}\left[\int_{t}^{\tau} \mathrm{e}^{-r(s-t)} f\left(X_{s}\right) \mathrm{d} s\right] .
\end{aligned}
$$

This work is inspired by Pedersen and Peskir [5], who verified the equivalence of problems (2) and (4) for $f(x)=x$, where $X_{t}$ is an Ornstein-Uhlenbeck process. Note that we do not consider the case $f(x)=x$, so our approach to deal with the optimal problem is different from that of Pedersen and Peskir. Moreover, our work naturally explore the explicit solutions of the new optimal problem (4) for a larger class of reward functions and underlying processes $X_{t}$.

The paper is organized as follows. In Section 2, the explicit value function and optimal stopping time are derived for a general Markov process along with the condition for the reward functions. Section 3 discusses some applications to diffusion, which include Brownian motion with drift, geometric Brownian motion, and the Ornstein-Uhlenbeck process. Section 4 displays some concrete examples of Lévy processes with jumps. In Section 5, we will link the outcomes with other optimal problems such that explicit solutions for the new problems can also be feasible to a general Markov process with a large class of reward functions. Finally, concluding remarks are given in Section 6.

\section{Optimal Rule for Continuous-Time Markov Processes}

For a Markov process $X_{t}$, the infinitesimal generator of $X_{t}$ is defined as

$$
\mathscr{G} g(x)=\lim _{t \searrow 0} \frac{\mathrm{E}^{x} g\left(X_{t}\right)-g(x)}{t}
$$

where $g(x)$ is twice continuous differentiable. In the diffusion case, namely,

$$
\mathrm{d} X_{t}=a\left(X_{t}\right) \mathrm{d} t+\sigma\left(X_{t}\right) \mathrm{d} W_{t}
$$

where $W_{t}$ is a standard Brownian motion, $a(x)>0, \sigma(x) \neq 0$, the infinitesimal generator is equivalent to

$$
\mathscr{G} g(x)=a(x) \frac{\mathrm{d} g}{\mathrm{~d} x}+\frac{\sigma^{2}(x)}{2} \frac{\mathrm{d}^{2} g}{\mathrm{~d} x^{2}} .
$$

In the case of Lévy process with jumps, driven by the equation

$$
\mathrm{d} X_{t}=a\left(X_{t-}\right) \mathrm{d} t+\sigma\left(X_{t-}\right) \mathrm{d} W_{t}+c\left(X_{t-}\right) \mathrm{d} N_{t},
$$

where $N_{t}$ is a homogeneous Poisson process, $a(x)>0$, $\sigma(x) \neq 0, c(x)>0$, the infinitesimal generator is

$$
\begin{aligned}
\mathscr{G} g(x)= & a(x) \frac{\mathrm{d} g}{\mathrm{~d} x}+\frac{\sigma^{2}(x)}{2} \frac{\mathrm{d}^{2} g}{\mathrm{~d} x^{2}} \\
& +\int(g(x+c(y))-g(x)) \Pi(\mathrm{d} y),
\end{aligned}
$$

where $\Pi(\cdot)$ is the Lévy measure (for jump diffusion and its generators, e.g., we can refer to Gihman and Skorohod [6]).

First, we make the assumption about the reward function.

Assumption 1. The reward function $f(x)$ is nonincreasing, concave, and twice continuous differentiable; that is, $\mathrm{d} f(x) / \mathrm{d} x \leq 0, \mathrm{~d}^{2} f(x) / \mathrm{d} x^{2} \leq 0$, and $f(x)$ is $C^{2}$.

Under Assumption 1, we present the explicit solutions for general continuous-time Markov processes.

Theorem 2. For a Markov process with infinitesimal generator $\mathscr{G}$, let $g(x)$ be the solution of

$$
\mathscr{G}(x)=r g(x)
$$

satisfying

$$
\begin{aligned}
\lim _{x \rightarrow \infty} g(x) & =0, \\
\frac{\mathrm{d} g(x)}{\mathrm{d} x} & \leq 0, \\
\frac{\mathrm{d}^{2} g(x)}{\mathrm{d} x^{2}} & \geq 0 .
\end{aligned}
$$

Given the reward function $f(x)$ in Assumption 1, if there exists a point $x^{*}$ such that

$$
\left.\frac{\mathrm{d} f(x)}{\mathrm{d} x}\right|_{x=x^{*}}=\left.\frac{f\left(x^{*}\right)}{g\left(x^{*}\right)} \frac{\mathrm{d} g(x)}{\mathrm{d} x}\right|_{x=x^{*}}, \quad f\left(x^{*}\right)>0,
$$

then the optimal problem

$$
v(x)=\sup _{\tau} \mathrm{E}^{x}\left[\mathrm{e}^{-r(\tau-t)} f\left(X_{\tau}\right)\right]
$$

has an explicit expression for the value function

$$
v(x)= \begin{cases}f(x), & x \leq x^{*}, \\ \frac{f\left(x^{*}\right)}{g\left(x^{*}\right)} g(x), & x \geq x^{*} .\end{cases}
$$

The optimal stopping time is

$$
\tau^{*}=\inf \left\{s \geq t: X_{s} \leq x^{*}\right\} .
$$

Proof. Define a function

$$
h(x)=\frac{f\left(x^{*}\right)}{g\left(x^{*}\right)} g(x)-f(x),
$$


and then we get

$$
\begin{aligned}
& h(x)=0, \\
& \frac{\mathrm{d} h(x)}{\mathrm{d} x}=\frac{f\left(x^{*}\right)}{g\left(x^{*}\right)} \frac{\mathrm{d} g(x)}{\mathrm{d} x}-\frac{\mathrm{d} f(x)}{\mathrm{d} x}=0 \text { iff } x=x^{*}, \\
& \frac{\mathrm{d}^{2} h(x)}{\mathrm{d} x^{2}}=\frac{f\left(x^{*}\right)}{g\left(x^{*}\right)} \frac{\mathrm{d}^{2} g(x)}{\mathrm{d} x^{2}}-\frac{\mathrm{d}^{2} f(x)}{\mathrm{d} x^{2}} \geq 0 .
\end{aligned}
$$

Hence, $h(x)$ attains the minimum at $x=x^{*},\left(f\left(x^{*}\right) /\right.$ $\left.g\left(x^{*}\right)\right) g(x) \geq f(x)$. So we have the inequality

$$
\mathrm{E}^{x} \mathrm{e}^{-r(\tau-t)} f\left(X_{\tau}\right) \leq \mathrm{E}^{x} \mathrm{e}^{-r(\tau-t)} v\left(X_{\tau}\right) .
$$

The value function $v(x)$ is $C^{1}, v^{\prime \prime}(x)$ exists, and is continuous except at $x^{*}$. By Itô's formula,

$$
\begin{aligned}
\mathrm{e}^{-r u} v\left(X_{u}\right)= & \mathrm{e}^{-r t} v\left(X_{t}\right)+\text { local martingale } \\
& +\int_{t}^{u} \mathrm{e}^{-r s}(\mathscr{G}-r) v\left(X_{s}\right) \mathrm{d} s,
\end{aligned}
$$

for any time $u>t$, where $\mathscr{G}$ is the infinitesimal generator of the process $X_{t}$.

Because the value function $v(x)$ is bounded, the local martingale term on the right-hand side of (21) is also bounded (all the other terms in (21) are clearly bounded), implying that it is in fact a martingale with zero expectation. Hence, by the optimal sampling theorem, we have, for any stopping time $\tau$,

$$
\begin{aligned}
\mathrm{E}^{x} \mathrm{e}^{-r(\tau-t)} v\left(X_{\tau}\right)= & v\left(X_{0}\right) \\
& +\mathrm{E}^{x} \int_{t}^{\tau} \mathrm{e}^{-r(s-t)}(\mathscr{G}-r) v\left(X_{s}\right) \mathrm{d} s .
\end{aligned}
$$

(a) When $x \leq x^{*}, v(x)=f(x)$, we claim that

$$
(\mathscr{G}-r) v(x)=(\mathscr{G}-r) f(x) \leq 0 .
$$

For diffusion,

$$
(\mathscr{G}-r) f(x)=a(x) \frac{\mathrm{d} f}{\mathrm{~d} x}+\frac{\sigma^{2}(x)}{2} \frac{\mathrm{d}^{2} f}{\mathrm{~d} x^{2}}-r f(x),
$$

$a(x)>0, \mathrm{~d} f(x) / \mathrm{d} x \leq 0, \mathrm{~d}^{2} f(x) / \mathrm{d} x^{2} \leq 0, f(x) \geq f\left(x^{*}\right)>0$, and the inequality in (23) holds naturally. For Lévy processes with jumps,

$$
\begin{aligned}
(\mathscr{G}-r) f(x)= & a(x) \frac{\mathrm{d} f}{\mathrm{~d} x}+\frac{\sigma^{2}(x)}{2} \frac{\mathrm{d}^{2} f}{\mathrm{~d} x^{2}} \\
& +\int(f(x+c(y))-f(x)) \Pi(\mathrm{d} y) \\
& -r f(x) .
\end{aligned}
$$

As $c(y)>0$ and $f(x)$ is decreasing on states, then $\int(f(x+$ $c(y))-f(x)) \Pi(\mathrm{d} y)<0$. (b) When $x \geq x^{*}, v(x)=\left(f\left(x^{*}\right) / g\left(x^{*}\right)\right) g(x)$, the function $g(x)$ is the solution of

$$
\mathscr{G} g(x)=r g(x)
$$

so $(\mathscr{G}-r) v(x)=0$.

Therefore, from (a) and (b), we have $(\mathscr{G}-r) v(x) \leq 0$, and the equality holds for $x \geq x^{*}$. It turns to be

$$
\begin{aligned}
\mathrm{E}^{x} \mathrm{e}^{-r(\tau-t)} f\left(X_{\tau}\right) \leq & \mathrm{E}^{x} \mathrm{e}^{-r(\tau-t)} v\left(X_{\tau}\right) \\
= & v\left(X_{0}\right) \\
& +\mathrm{E}^{x} \int_{t}^{\tau} \mathrm{e}^{-r(s-t)}(\mathscr{G}-r) v\left(X_{s}\right) \mathrm{d} s \\
\leq & v(x) .
\end{aligned}
$$

From which we can see that $v(x)$ is an upper bound for the value starting from $X_{0}=x$. This bound is achieved when $\tau=$ $\tau^{*}$. When the starting state $x$ is smaller than $x^{*}$, the optimal stopping time is $\tau^{*}=t$. That is, $\sup _{\tau} \mathrm{E}^{x} \mathrm{e}^{-r(\tau-t)} f\left(X_{\tau}\right)=$ $\mathrm{E}^{x} f\left(X_{t}\right)=v(x)$ as it reaches its upper bound. If the starting state is greater than the point $x^{*}$, it must wait until $\tau^{*}$, which is the first hitting time to the point $x^{*}$. At the stopping time $\tau^{*}, f\left(X_{\tau^{*}}\right)=v\left(X_{\tau^{*}}\right)$, and $\int_{t}^{\tau^{*}} \mathrm{e}^{-r(s-t)}(\mathscr{G}-r) v\left(X_{s}\right) \mathrm{d} s=0$, so $\mathrm{E}^{x} \mathrm{e}^{-r\left(\tau^{*}-t\right)} f\left(X_{\tau^{*}}\right)=v(x)$, reaching its upper bound.

Remark 3. Actually, for all diffusion, Theorem 2 holds for the drift term satisfying $a(x)<0, a^{\prime}(x)<r$. We calculate that

$$
\begin{aligned}
& \left(a(x) \frac{\mathrm{d} f(x)}{\mathrm{d} x}-r f(x)\right)^{\prime} \\
& =a^{\prime}(x) f^{\prime}(x)+a(x) f^{\prime \prime}(x)-r f^{\prime}(x) \geq 0,
\end{aligned}
$$

so for $x \leq x^{*}$,

$$
\begin{gathered}
a(x) f^{\prime}(x)-r f(x) \leq a\left(x^{*}\right) f^{\prime}\left(x^{*}\right)-r f\left(x^{*}\right) \\
=\frac{f\left(x^{*}\right)}{g\left(x^{*}\right)}\left(a\left(x^{*}\right) g^{\prime}\left(x^{*}\right)-r g\left(x^{*}\right)\right) .
\end{gathered}
$$

As $g\left(x^{*}\right)$ satisfies $a\left(x^{*}\right) g^{\prime}\left(x^{*}\right)+\left(\sigma^{2}\left(x^{*}\right) / 2\right) g^{\prime \prime}\left(x^{*}\right)-r g\left(x^{*}\right)=$ 0 , we can arrive at

$$
\begin{aligned}
(\mathscr{G}-r) f(x)= & a(x) f^{\prime}(x)+\frac{\sigma^{2}(x)}{2} f^{\prime \prime}(x)-r f(x) \\
\leq & -\frac{\sigma^{2}\left(x^{*}\right)}{2} \frac{f\left(x^{*}\right)}{g\left(x^{*}\right)} g^{\prime \prime}\left(x^{*}\right) \\
& +\frac{\sigma^{2}(x)}{2} f^{\prime \prime}(x) \leq 0 .
\end{aligned}
$$

The rest of the proof is the same as that in Theorem 2.

However, the condition for Lévy process will be more complicated. In order to have a uniform style, we restrict to the case $a(x)>0$. Moreover, we can see from (19) that if the point $x^{*}$ exists, then it is unique. Next we present results on some classical Markov processes as applications of Theorem 2. 


\section{Diffusion}

3.1. Brownian Motion with Drift. For Brownian motion with drift $\mu>0$, and variance $\sigma^{2}(\sigma \neq 0)$, namely, the process is driven by the SDE

$$
\begin{aligned}
\mathrm{d} X_{t} & =\sigma \mathrm{d} W_{t}+\mu \mathrm{d} t, \\
X_{0} & =x>0,
\end{aligned}
$$

by using Theorem 2, we get the following proposition.

Proposition 4. Let $f(x)$ be the function in Assumption 1; if there exists a point $x^{*}$ such that

$$
\left.\frac{\mathrm{d} f(x)}{\mathrm{d} x}\right|_{x=x^{*}}=-\left(\sqrt{\frac{\mu^{2}}{\sigma^{4}}+\frac{2 r}{\sigma^{2}}}+\frac{\mu}{\sigma^{2}}\right) f\left(x^{*}\right),
$$

$$
f\left(x^{*}\right)>0
$$

then the value function $v(x)$ has the form

$$
\begin{aligned}
& v(x)
\end{aligned}
$$

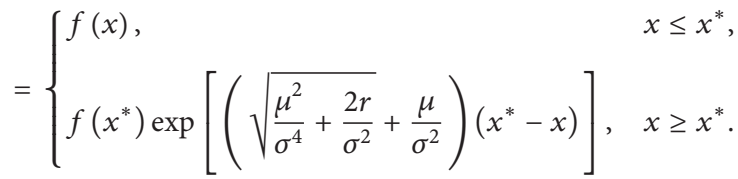

The optimal stopping time is

$$
\tau^{*}=\inf \left\{s \geq t: X_{s} \leq x^{*}\right\} .
$$

Proof. The infinitesimal generator for Brownian motion with drift is $\mathscr{G}=\mu(\mathrm{d} / \mathrm{d} x)+\left(\sigma^{2} / 2\right)\left(\mathrm{d}^{2} / \mathrm{d} x^{2}\right)$. It is well known that the ordinary differential equation (ODE) of $\mathscr{G} g(x)=r g(x)$ has two linearly independent solutions

$$
\begin{aligned}
& \phi(x)=\exp \left[x\left(\sqrt{\frac{\mu^{2}}{\sigma^{4}}+\frac{2 r}{\sigma^{2}}-\frac{\mu}{\sigma^{2}}}\right)\right], \\
& \varphi(x)=\exp \left[-x\left(\sqrt{\left.\left.\frac{\mu^{2}}{\sigma^{4}}+\frac{2 r}{\sigma^{2}}+\frac{\mu}{\sigma^{2}}\right)\right] .}\right.\right.
\end{aligned}
$$

So $g(x)=c 1 \phi(x)+c 2 \varphi(x)$ ( $c 1$ and $c 2$ are constants). Considering the boundary condition $\lim _{x \rightarrow \infty} g(x)=0$ and $g^{\prime}(x) \leq 0, g^{\prime \prime}(x) \geq 0$, then $c 1$ must be equal to zero, $g(x)=$ $c 2 \varphi(x)$, and $c 2>0$. Equation (13) in Theorem 2 tells us that the point $x^{*}$ is determined by

$$
\begin{aligned}
f^{\prime}\left(x^{*}\right) & =\frac{f\left(x^{*}\right)}{g\left(x^{*}\right)} g^{\prime}\left(x^{*}\right) \\
& =-\left(\sqrt{\frac{\mu^{2}}{\sigma^{4}}+\frac{2 r}{\sigma^{2}}}+\frac{\mu}{\sigma^{2}}\right) f\left(x^{*}\right),
\end{aligned}
$$

since $g^{\prime}(x) / g(x)=-\sqrt{\mu^{2} / \sigma^{4}+2 r / \sigma^{2}}-\mu / \sigma^{2}$. Thus, the expression for the value function $v(x)$ is easily obtained by (15).

As the simplest example, we take the reward function $f(x)=1-x$ and we take the parameters $\mu=1, \sigma=\sqrt{2}$, and $r=1$. Then, the problem $v(x)=\sup _{\tau} \mathrm{E}^{x}\left[\mathrm{e}^{-(\tau-t)}\left(1-X_{\tau}\right)\right]$ has the solution

$$
v(x)= \begin{cases}1-x, & x \leq \frac{3}{2}-\frac{\sqrt{5}}{2} \\ \left(\frac{\sqrt{5}}{2}-\frac{1}{2}\right) \exp \left[-\left(\frac{\sqrt{5}}{2}+\frac{1}{2}\right) x+\frac{\sqrt{5}}{2}-\frac{1}{2}\right], & x \geq \frac{3}{2}-\frac{\sqrt{5}}{2}\end{cases}
$$

The optimal stopping time is

$$
\tau^{*}=\inf \left\{s \geq t: X_{s} \leq \frac{3}{2}-\frac{\sqrt{5}}{2}\right\} .
$$

We draw the value function and the point $x^{*}$ in Figure 1.

3.2. Geometric Brownian Motion. As for geometric Brownian motion, that is, the process $X_{t}$ is driven by

$$
\mathrm{d} X_{t}=X_{t}\left(\sigma \mathrm{d} W_{t}+\mu \mathrm{d} t\right), \quad X_{0}=x>0,
$$

we derive the following proposition.
Proposition 5. Let $f(x)$ be the function in Assumption 1; if there exists a point $x^{*}$ such that

$$
\left.\frac{\mathrm{d} f(x)}{\mathrm{d} x}\right|_{x=x^{*}}=\beta \frac{f\left(x^{*}\right)}{x^{*}}, \quad f\left(x^{*}\right)>0,
$$

where $\beta=1 / 2-\mu / \sigma^{2}-\sqrt{\left(1 / 2-\mu / \sigma^{2}\right)^{2}+2 r / \sigma^{2}}$, then the value function $v(x)$ has the form

$$
v(x)= \begin{cases}f(x), & x \leq x^{*} \\ f\left(x^{*}\right)\left(\frac{x}{x^{*}}\right)^{\beta}, & x \geq x^{*}\end{cases}
$$

The optimal stopping time is

$$
\tau^{*}=\inf \left\{s \geq t: X_{s} \leq x^{*}\right\} .
$$




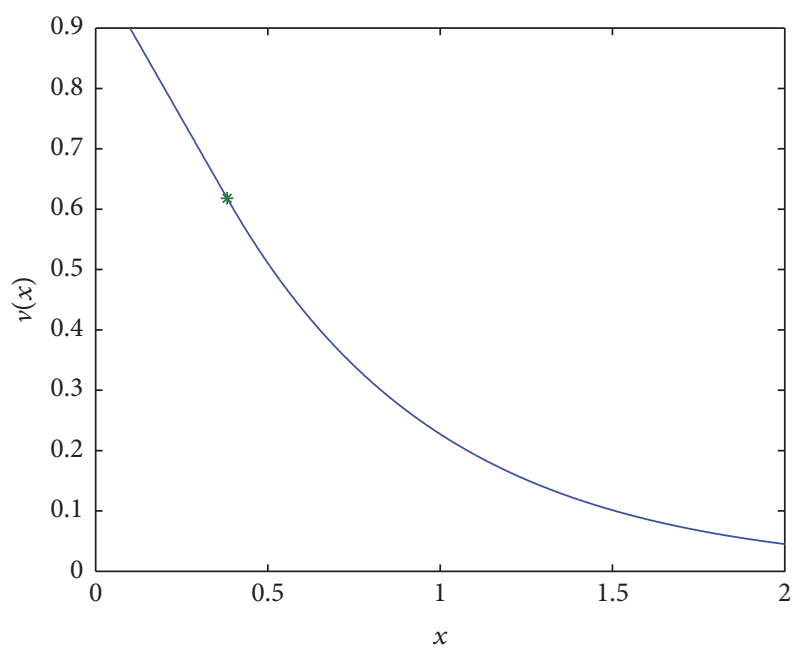

Figure 1: Plot of the value function and the point $x^{*}\left(x^{*} \simeq 0.3820\right)$ for Brownian motion with parameters $\mu=1, \sigma=\sqrt{2}$, and $r=1$.

Proof. The infinitesimal generator for geometric Brownian motion is $\mathscr{G}=\mu x(\mathrm{~d} / \mathrm{d} x)+\left(\sigma^{2} x^{2} / 2\right)\left(\mathrm{d}^{2} / \mathrm{d} x^{2}\right)$. The associated ODE of $\mathscr{G} g(x)=r g(x)$ has two linearly independent solutions $\phi(x)^{\beta^{\prime}}$ and $\varphi(x)^{\beta}$, where $\beta^{\prime}=1 / 2-$ $\mu / \sigma^{2}+\sqrt{\left(1 / 2-\mu / \sigma^{2}\right)^{2}+2 r / \sigma^{2}}$ and $\beta=1 / 2-\mu / \sigma^{2}-$ $\sqrt{\left(1 / 2-\mu / \sigma^{2}\right)^{2}+2 r / \sigma^{2}}$. By using the boundary conditions of Theorem 2, we find that $g(x)=c \varphi(x)^{\beta}$, where $c$ is a constant bigger than zero. Then, by substituting $g(x)$ in Theorem 2 , we deduce the value function and the point $x^{*}$.

Remark 6. If the volatility $\sigma$ is equal to zero, then the drift $\mu$ should be smaller than zero. Or the problem cannot be solved by Theorem 2 . We take the reward function $f(x)=1-x$ as an illustration. For $\sigma=0$ and $\mu<0$, from Theorem 2, the solution for the optimal value function is

$$
v(x)= \begin{cases}1-x, & x \leq \frac{r}{r-\mu}, \\ \frac{-\mu}{r-\mu}\left(\frac{r x-\mu x}{r}\right)^{r / \mu}, & x \geq \frac{r}{r-\mu} .\end{cases}
$$

The optimal stopping time is

$$
\tau^{*}=\inf \left\{t: X_{t} \leq \frac{r}{r-\mu}\right\} .
$$

If $\sigma=0$ and $\mu>0, \mathrm{~d} X_{s}=\mu X_{s} \mathrm{~d} s$, that is, $X_{s}=x e^{\mu(s-t)}$, for any time $s \geq t$. By direct computation, we find that

$$
\begin{aligned}
v(x) & =\sup _{\tau} \mathrm{E}^{x}\left[\mathrm{e}^{-r(\tau-t)}\left(1-x e^{\mu(\tau-t)}\right)\right] \\
& = \begin{cases}0, & x \geq 1, \\
1-x, & x \leq 1,\end{cases}
\end{aligned}
$$

with the optimal stopping time

$$
\tau^{*}=\inf \left\{t: X_{t} \leq 1\right\} \text {. }
$$

When the starting state is smaller than 1 , it should take action at once. When the starting state is greater than 1 , it will never invest, because the process is increasing, and it never reaches its optimal invest time. But, in this case, the point $x^{*}=1$ is not determined by (13). Because the left side is -1 and the right side is 0 if we replace $f(x)=1-x$ and $x^{*}=1$ in (13).

Taking parameters $\mu=1, \sigma=\sqrt{2}$, and $r=1$, the value function for the reward function $f(x)=1-x$ is

$$
v(x)= \begin{cases}1-x, & x \leq \frac{1}{2} \\ \frac{1}{4 x}, & x \geq \frac{1}{2}\end{cases}
$$

The optimal stopping time is

$$
\tau^{*}=\inf \left\{t: X_{t} \leq \frac{1}{2}\right\} .
$$

This problem's solution is the key to finding the optimum

$$
v(x)=\sup _{\tau} \mathrm{E}^{x}\left[\mathrm{e}^{-r \tau}\left(X_{\tau}^{1}-X_{\tau}^{2}\right)\right],
$$

which has many applications in finance, and it has been considered for geometric Brownian motion from different points of view in a variety of articles. Let us just mention [14, 7-9].

3.3. Ornstein-Uhlenbeck Process. In this subsection we consider the optimal stopping problem when $X$ is an OrnsteinUhlenbeck process,

$$
\mathrm{d} X_{t}=\sigma \mathrm{d} W_{t}-\mu X_{t} \mathrm{~d} t, \quad X_{0}=x .
$$

Proposition 7. Let $f(x)$ be as in Assumption 1; if there exists a point $x^{*}$ such that

$$
\left.\frac{\mathrm{d} f(x)}{\mathrm{d} x}\right|_{x=x^{*}}=-f\left(x^{*}\right) \frac{G_{r / \mu+1}\left(x^{*}\right)}{G_{r / \mu}\left(x^{*}\right)}, \quad f\left(x^{*}\right)>0,
$$

then the value function $v(x)$ has the form

$$
v(x)= \begin{cases}f(x), & x \leq x^{*} \\ f\left(x^{*}\right) \frac{G_{r / \mu}(x)}{G_{r / \mu}\left(x^{*}\right)}, & x \geq x^{*}\end{cases}
$$

where

$$
G_{r / \mu}(x)=\int_{0}^{\infty} s^{r / \mu-1} \exp \left(-x s-\frac{\sigma^{2} s^{2}}{4 \mu}\right) \mathrm{d} s .
$$

The optimal stopping time is

$$
\tau^{*}=\inf \left\{s \geq t: X_{s} \leq x^{*}\right\} .
$$

Proof. For the Ornstein-Uhlenbeck process, the infinitesimal generator is $\mathscr{G}=-\mu x(\mathrm{~d} / \mathrm{d} x)+\left(\sigma^{2} / 2\right)\left(\mathrm{d}^{2} / \mathrm{d} x^{2}\right)$. The ODE of $\mathscr{G} g(x)=r g(x)$ has two linearly independent solutions

$$
\begin{aligned}
& F_{r / \mu}(x)=\int_{0}^{\infty} s^{r / \mu-1} \exp \left(x s-\frac{\sigma^{2} s^{2}}{4 \mu}\right) \mathrm{d} s, \\
& G_{r / \mu}(x)=\int_{0}^{\infty} s^{r / \mu-1} \exp \left(-x s-\frac{\sigma^{2} s^{2}}{4 \mu}\right) \mathrm{d} s .
\end{aligned}
$$


The solution satisfying the boundary conditions in Theorem 2 is $g(x)=c G_{r / \mu}(x)$, where $c$ is a constant bigger than zero. Thanks to the relations

$$
\begin{aligned}
G_{r / \mu}(x) & =\frac{\mu x}{r} G_{r / \mu+1}(x)+\frac{\sigma^{2}}{2 r} G_{r / \mu+2}(x), \\
\frac{\mathrm{d} G_{r / \mu}(x)}{\mathrm{d} x} & =-G_{r / \mu+1}(x), \\
\frac{\mathrm{d}^{2} G_{r / \mu}(x)}{\mathrm{d} x} & =G_{r / \mu+2}(x),
\end{aligned}
$$

we obtain the value function and the point $x^{*}$.

Regarding the reward function $f(x)=1-x$, if there exists a point $x^{*}$ such that $f\left(x^{*}\right)=1-x^{*}>0$ and $G_{r / \mu+1}\left(x^{*}\right)(1-$ $\left.x^{*}\right)=G_{r / \mu}\left(x^{*}\right)$, the problem $v(x)$ has the form

$$
v(x)= \begin{cases}1-x, & x \leq x^{*}, \\ \left(1-x^{*}\right) \frac{G_{r / \mu}(x)}{G_{r / \mu}\left(x^{*}\right)}, & x \geq x^{*} .\end{cases}
$$

The optimal stopping time is

$$
\tau^{*}=\inf \left\{s \geq t: X_{s} \leq x^{*}\right\} .
$$

When parameters are chosen as $\mu=1, \sigma=\sqrt{2}$, and $r=1$, the point $x^{*}$ is the solution of

$$
G_{2}(x)(1-x)=G_{1}(x) .
$$

Thanks to

$$
\begin{aligned}
G_{1}(x) & =\int_{0}^{\infty} \exp \left(-x s-\frac{s^{2}}{2}\right) \mathrm{d} s \\
& =\exp \left(\frac{x^{2}}{2}\right) \int_{x}^{\infty} \exp \left(-\frac{s^{2}}{2}\right) \mathrm{d} s, \\
G_{2}(x) & =1-x G_{1}(x)
\end{aligned}
$$

$G_{1}(x)$ and $G_{2}(x)$ can be reduced to expressions of gammainc $\left(x^{2} / 2,1 / 2\right)$ in Matlab. By numerical calculation, we get the point $x^{*} \simeq-0.1628$ and we show the value function in Figure 2.

\section{Lévy Processes with Jumps}

4.1. The Jump Diffusion. Jump diffusion processes are processes of the form

$$
\mathrm{d} X_{t}=\sigma \mathrm{d} W_{t}+\mu \mathrm{d} t+\mathrm{d}\left(\sum_{i=1}^{N_{t}} Y_{i}\right), \quad X_{0}=x
$$

They are studied in Kou and Wang [10], as regards first passage times. Here, $N_{t}$ is a homogeneous Poisson process with intensity rate $\lambda>0$, drift $\mu>0$, and volatility $\sigma>0$, and the jump sizes $\left\{Y_{1}, Y_{2}, \ldots\right\}$ are independent and identically distributed random variables. We also assume

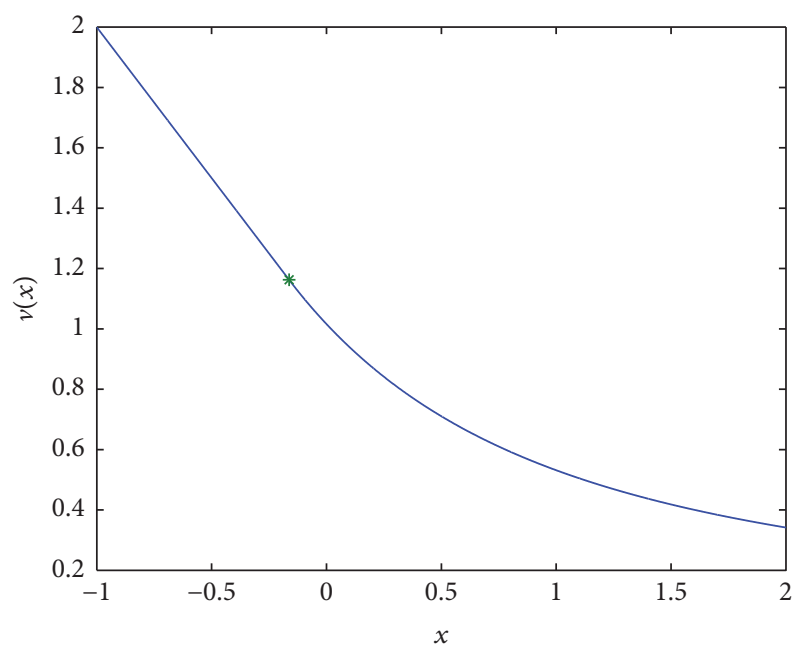

FIgURE 2: The value function and the point $x^{*} \simeq-0.1628$ for the Ornstein-Uhlenbeck process with parameters $\mu=1, \sigma=\sqrt{2}$, and $r=1$, with regard to the reward function $f(x)=1-x$.

that the standard Brownian motion $W_{t}$ is independent of $\left\{Y_{1}, Y_{2}, \ldots\right\}$. The common density of $Y$ is given by

$$
p_{Y}(y)=\eta \mathrm{e}^{-\eta y}
$$

where $\eta>0$.

Proposition 8. Regarding the reward function $f(x)$ in Assumption 1, if there exists a point $x^{*}$ such that

$$
\left.\frac{\mathrm{d} f(x)}{\mathrm{d} x}\right|_{x=x^{*}}=\beta f\left(x^{*}\right), \quad f\left(x^{*}\right)>0,
$$

where $\beta<0$ is the negative root of

$$
\frac{\sigma^{2}}{2} y^{2}+\mu y+\lambda \frac{y}{\eta-y}-r=0,
$$

then the value function $v(x)$ has the form

$$
v(x)= \begin{cases}f(x), & x \leq x^{*}, \\ f\left(x^{*}\right) \exp \left(\beta x-\beta x^{*}\right), & x \geq x^{*} .\end{cases}
$$

The optimal stopping time is

$$
\tau^{*}=\inf \left\{s \geq t: X_{s} \leq x^{*}\right\} .
$$

Proof. In this case

$$
\begin{aligned}
(\mathscr{G}-r) v(x)= & \mu \frac{\mathrm{d} v(x)}{\mathrm{d} x}+\frac{\sigma^{2}}{2} \frac{\mathrm{d}^{2} v(x)}{\mathrm{d} x^{2}} \\
& +\lambda \int_{0}^{\infty}[v(x+y)-v(x)] \eta \mathrm{e}^{-\eta y} \mathrm{~d} y \\
& -r v(x) .
\end{aligned}
$$


(a) When $x \leq x^{*}, v(x+y)-v(x) \leq 0$ for every $y>$ $0, \mathrm{~d} f(x) / \mathrm{d} x \leq 0, \mathrm{~d}^{2} f(x) / \mathrm{d} x^{2} \leq 0$, for $\mu>0$, and $(\mathscr{G}-r) v\left(X_{s}\right) \leq 0$.

(b) When $x \geq x^{*},(\mathscr{G}-r) v(x)=\left((1 / 2) \sigma^{2} \beta^{2}+\mu \beta+\right.$ $\lambda(\beta /(\eta-\beta))-r) f\left(x^{*}\right) \exp \left(\beta x-\beta x^{*}\right)=0$. Since $(\mathscr{G}-r) v(x) \leq 0$ for all $x$, the rest of the proof is the same as in Theorem 2.

Remark 9. We assume that $\mu>0$ as Kou and Wang [10] do, and we do not know whether the results hold for $\mu \leq 0$.

Let $H(y)$ be defined as $H(y)=(1 / 2) \sigma^{2} y^{2}+\mu y+\lambda(y /(\eta-$ $y)$ ), and it has three roots, $y_{1}=0, y_{2}=\eta / 2-\mu / \sigma^{2}+$ $\sqrt{\left(\eta / 2+\mu / \sigma^{2}\right)^{2}+2 \lambda / \sigma^{2}}>0$, and $y_{3}=\eta / 2-\mu / \sigma^{2}-$ $\sqrt{\left(\eta / 2+\mu / \sigma^{2}\right)^{2}+2 \lambda / \sigma^{2}}<0$.

Next, we prove that the equation $H(y)=r$ for all $r>0$ has exactly a negative root $\beta$.

$$
\begin{aligned}
& H^{\prime}(y)=\sigma^{2} y+\mu+\frac{\lambda \eta}{(\eta-y)^{2}}, \\
& H^{\prime \prime}(y)=\sigma^{2}+\frac{2 \lambda \eta}{(\eta-y)^{3}},
\end{aligned}
$$

so $H(y)$ is increased and convex on the interval $(0, \eta)$ with $H(0)=0$ and $H(\eta-)=\infty$, and there is exactly one root for $H(y)=r$. Furthermore, since $H(\eta+)=-\infty, H(\infty)=\infty$, there is at least one root on $(\eta, \infty)$. Similarly, there is at least one root on $(-\infty, 0)$, as $H(-\infty)=\infty$ and $H(0)=0$. But the equation $H(y)=r$ is actually a polynomial equation with degree three; therefore, it can have at most three real roots. It follows that, on each interval, $(-\infty, 0)$ and $(\eta, \infty)$, there is exactly one root.

As an example, we take the reward function $f(x)=1-$ $x$ again. From Proposition $8, x^{*}=1+1 / \beta$, where $\beta$ is the negative root of

$$
\frac{1}{2} \sigma^{2} y^{2}+\mu y+\frac{\lambda y}{\eta-y}-r=0
$$

and the value function $v(x)$ has the form

$$
v(x)= \begin{cases}1-x, & x \leq x^{*}, \\ \left(1-x^{*}\right) \exp \left(\beta x-\beta x^{*}\right), & x \geq x^{*} .\end{cases}
$$

The optimal stopping time is

$$
\tau^{*}=\inf \left\{s \geq t: X_{s} \leq x^{*}\right\} .
$$

If the parameters are $\mu=1, \sigma=\sqrt{2}, r=1, \lambda=1$, and $\eta=$ 1.5 , we show the value function and the point $x^{*}$ in Figure 3 . Numerical calculation gets the negative solution for $\beta$ to be $\beta \simeq-1.8421$, and the point $x^{*} \simeq 0.4571$. Compared with the case of Brownian motion with drift, the decision point $x^{*}$ results larger after adding the jump process. Intuitively, as risk

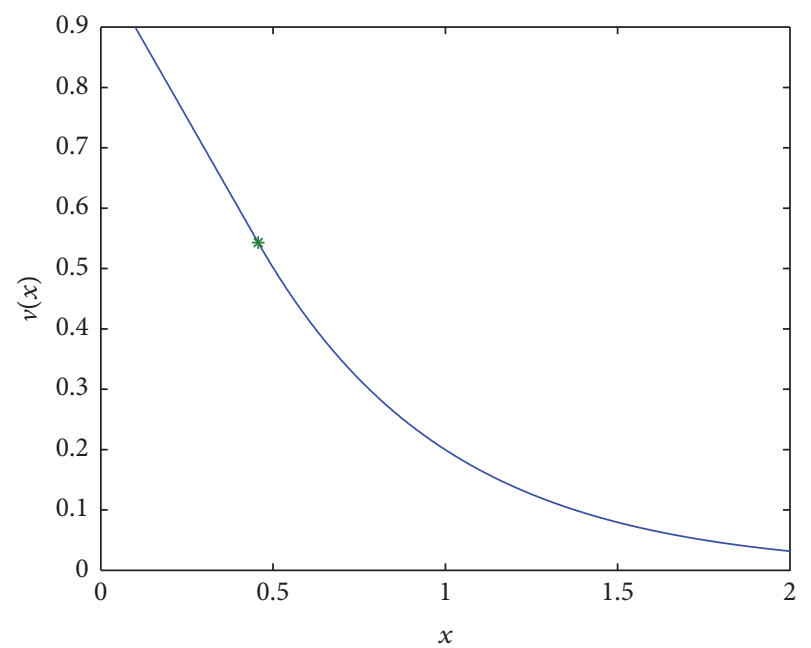

FIGURE 3: The value function and the point $x^{*}\left(x^{*} \simeq 0.4571\right)$ for the jump diffusion model with parameters $\mu=1, \sigma=\sqrt{2}, r=1, \lambda=1$, and $\eta=1.5$.

in the system is increasing, the investors are intent to wait and observe for a longer time, so that the optimal stopping occurs later and the decision point gets greater.

The more general problem, when the common density of $Y$ is given by

$$
p_{Y}(y)=\sum_{i=1}^{m} p_{i} \eta_{i} \mathrm{e}^{-\eta_{i} y},
$$

where $0<\eta_{1}<\eta_{2}<\cdots<\eta_{m}<\infty$, and $\sum_{i=1}^{m} p_{i}=1$, has the following result.

Proposition 10. Let $f(x)$ be as in Assumption 1; if there exists a point $x^{*}$ such that

$$
\left.\frac{\mathrm{d} f(x)}{\mathrm{d} x}\right|_{x=x^{*}}=\beta f\left(x^{*}\right), \quad f\left(x^{*}\right)>0,
$$

where $\beta<0$ is the negative root of

$$
\frac{\sigma^{2}}{2} y^{2}+\mu y+\lambda \sum_{i=1}^{m} p_{i} \frac{y}{\eta_{i}-y}-r=0,
$$

then the value function $v(x)$ has the form

$$
v(x)= \begin{cases}f(x), & x \leq x^{*}, \\ f\left(x^{*}\right) \exp \left(\beta x-\beta x^{*}\right), & x \geq x^{*} .\end{cases}
$$

The optimal stopping time is

$$
\tau^{*}=\inf \left\{s \geq t: X_{s} \leq x^{*}\right\} .
$$

Remark 11. The equation $\left(\sigma^{2} / 2\right) y^{2}+\mu y+\lambda \sum_{i=1}^{m} p_{i}\left(y /\left(\eta_{i}-\right.\right.$ $y))-r=0$ has $(m+2)$ roots, which are all real and distinct. Indeed, it has $m+1$ positive roots, $\beta_{1}, \ldots, \beta_{m+1}$, and a negative $\operatorname{root} \beta$, as follows:

$$
-\infty<\beta<0<\beta_{1}<\cdots<\beta_{m+1}<\infty .
$$


Define $L(y)$ as

$$
L(y) \triangleq \frac{\sigma^{2}}{2} y^{2}+\mu y+\lambda \sum_{i=1}^{m} p_{i} \frac{y}{\eta_{i}-y},
$$

and we have $L(0)=0, L\left(\eta_{1}-\right)=\infty, L\left(\eta_{1}+\right)=-\infty, L\left(\eta_{2}-\right)=$ $\infty, \ldots, L\left(\eta_{m}+\right)=-\infty, L(\infty)=\infty$, and $L(y)$ is continuous and increasing in every interval $\left(0, \eta_{1}-\right),\left(\eta_{1}+\right.$, $\left.\eta_{2}-\right), \ldots,\left(\eta_{m}+, \infty\right)$. So it has $m+1$ positive roots, if it also has a negative root $\beta$ then the negative root is unique as it has at most $m+2$ real roots. The detailed proof can be found in Theorem 3.1 of Kou and Cai [11].

4.2. The Exponential Lévy-Type Stochastic Integral. The exponential Lévy-type stochastic integral $X_{t}$ is given by

$$
\begin{aligned}
& \frac{\mathrm{d} X_{t}}{X_{t-}}=\sigma \mathrm{d} W_{t}+\mu \mathrm{d} t+\mathrm{d}\left(\sum_{i=1}^{N_{t}}\left(\mathrm{e}^{Y_{i}}-1\right)\right) \\
& \\
& X_{0}=x>0 .
\end{aligned}
$$

Here, $N_{t}$ is a Poisson process with intensity rate $\lambda>0$, and $\mu$ and $\sigma$ are positive constants. The jump sizes $\left\{Y_{1}, Y_{2}, \ldots\right\}$ are independent and identically distributed random variables and independent of $W_{t}$. The common density of $Y$ is given by

$$
p_{Y}(y)=\eta \mathrm{e}^{-\eta y}
$$

where $\eta>1$ ensures that $X$ has a finite expectation.

Proposition 12. Given the reward function $f(x)$ in Assumption 1 , if there exists a point $x^{*}$ such that

$$
\left.\frac{\mathrm{d} f(x)}{\mathrm{d} x}\right|_{x=x^{*}}=\beta \frac{f\left(x^{*}\right)}{x^{*}}, \quad f\left(x^{*}\right)>0,
$$

where $\beta<0$ is the negative root of

$$
\frac{\sigma^{2}}{2} y^{2}(y-1)+\mu y+\lambda \frac{y}{\eta-y}-r=0,
$$

then the value function $v(x)$ has the form

$$
v(x)= \begin{cases}f(x), & x \leq x^{*}, \\ f\left(x^{*}\right)\left(\frac{x}{x^{*}}\right)^{\beta}, & x \geq x^{*} .\end{cases}
$$

The optimal stopping time is

$$
\tau^{*}=\inf \left\{s \geq t: X_{s} \leq x^{*}\right\} .
$$

Proof. In this case

$$
\begin{aligned}
(\mathscr{G}-r) v(x)= & \mu x \frac{\mathrm{d} v(x)}{\mathrm{d} x}+\frac{\sigma^{2} x^{2}}{2} \frac{\mathrm{d}^{2} v(x)}{\mathrm{d} x^{2}} \\
& +\lambda \int_{0}^{\infty}\left[v\left(x \mathrm{e}^{y}\right)-v(x)\right] \eta \mathrm{e}^{-\eta y} \mathrm{~d} y \\
& -r v(x)
\end{aligned}
$$

(a) When $x \leq x^{*}$, because $y \geq 0$ and $\mathrm{e}^{y} \geq 1$, then $x \mathrm{e}^{y} \geq x$ and $f\left(x \mathrm{e}^{y}\right) \leq f(x)$. Moreover, thanks to $\mu>0, \mathrm{~d} f(x) / \mathrm{d} x \leq 0, \mathrm{~d}^{2} f(x) / \mathrm{d} x^{2} \leq 0$, we have $(\mathscr{G}-r) v\left(X_{s}\right) \leq 0$.

(b) When $x \geq x^{*}$,

$(\mathscr{G}-r) v(x)$

$=\left[\frac{1}{2} \sigma^{2}\left(\beta^{2}-\beta\right)+\mu \beta+\lambda \frac{\beta}{\eta-\beta}-r\right] f\left(x^{*}\right)\left(\frac{x}{x^{*}}\right)^{\beta}$

$=0$.

Taking (a) and (b) together, we conclude that ( $\mathscr{G}-$ $r) v(x) \leq 0$ for every $x>0$. Hence, $v(x)$ is the upper bound for $\mathrm{E}^{x} \mathrm{e}^{-r(\tau-t)} f\left(X_{\tau}\right)$. At the optimal stopping time $\tau^{*}$, $\mathrm{E}^{x} \mathrm{e}^{-r\left(\tau^{*}-t\right)} f\left(X_{\tau^{*}}\right)=v(x)$, attaining the upper bound.

Remark 13. The equation $\left(\sigma^{2} / 2\right)(y-1)+\mu y+\lambda(y /(\eta-y))-$ $r=0$ has a unique negative $\operatorname{root} \beta$. This can be obtained proceeding as in Remark 11.

For the reward function $f(x)=1-x, \beta$ is the negative root of

$$
\frac{\sigma^{2}}{2} y(y-1)+\mu y+\frac{\lambda y}{\eta-y}-r=0,
$$

we find $x^{*}=\beta /(\beta-1)$, and the value function is

$$
v(x)= \begin{cases}1-x, & x \leq x^{*}, \\ \left(1-x^{*}\right)\left(\frac{x}{x^{*}}\right)^{\beta}, & x \geq x^{*} .\end{cases}
$$

The optimal stopping time is

$$
\tau^{*}=\inf \left\{s \geq t: X_{s} \leq x^{*}\right\} \text {. }
$$

For the choice of parameters $\mu=1, \sigma=\sqrt{2}, r=1, \lambda=1$, and $\eta=1.5$, we obtain $\beta \simeq-1.2020$ and the point $x^{*} \simeq 0.5459$, and we draw the graph of the value function in Figure 4. The point $x^{*}$ is bigger than the value concerning geometric Brownian motion, after adding the jump risk.

\section{Connection with the Other Optimal Problems}

As the reward function $f(x)$ is $C^{2}$ in Assumption 1, by Itô's formula,

$$
\begin{aligned}
\mathrm{e}^{-r(u-t)} f\left(X_{t}\right)= & f(x) \\
& +\int_{t}^{u} \mathrm{e}^{-r(s-t)}\left[\mathscr{G} f\left(X_{s}\right)-r f\left(X_{s}\right)\right] \mathrm{d} s
\end{aligned}
$$

+ local martingale. 


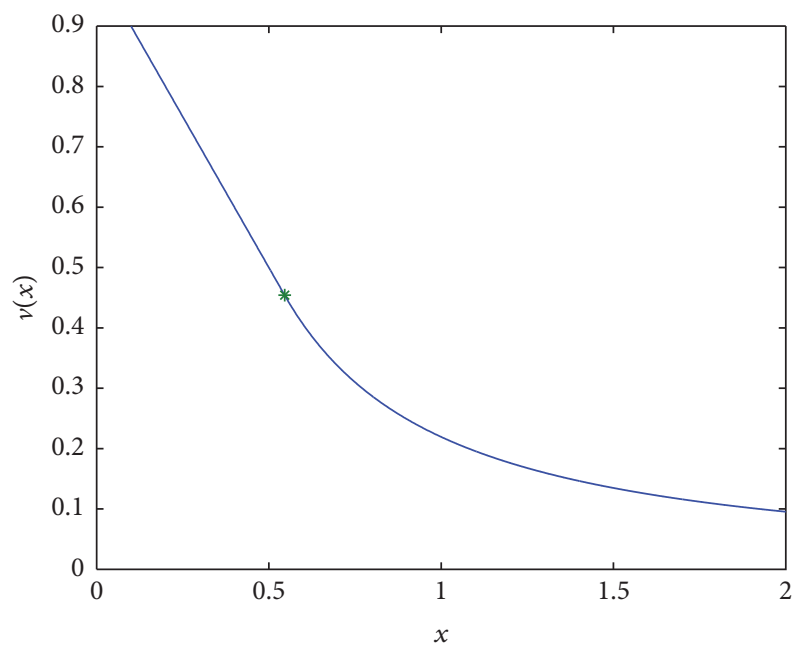

Figure 4: The value function and the point $x^{*}\left(x^{*} \simeq 0.5459\right)$ for $\mu=1, \sigma=\sqrt{2}, r=1, \lambda=1$, and $\eta=1.5$.

Assume that

$$
\mathscr{G} f\left(X_{s}\right)-r f\left(X_{s}\right)=\gamma f\left(X_{s}\right),
$$

where $\gamma \neq 0$ is a constant. Taking expectation in (90),

$$
\begin{aligned}
\mathrm{E}^{x} \mathrm{e}^{-r(u-t)} f\left(X_{u}\right)= & f(x)+\mathrm{E}^{x} \int_{t}^{u} \mathrm{e}^{-r(s-t)} \gamma f\left(X_{s}\right) \mathrm{d} s \\
& +\mathrm{E}^{x} \text { (local martingale) } .
\end{aligned}
$$

As assumed in the paper, $\mathrm{e}^{-r(u-t)} f\left(X_{t}\right)$ is bounded, so $\mathrm{E}^{x}($ local martingale $)=0$. For a stopping time $\tau$,

$$
\mathrm{E}^{x} \mathrm{e}^{-r(\tau-t)} f\left(X_{\tau}\right)=f(x)+\mathrm{E}^{x} \int_{t}^{\tau} \mathrm{e}^{-r(s-t)} \gamma f\left(X_{s}\right) \mathrm{d} s,
$$

from which we can see that if $\gamma>0$ the problem $\sup _{\tau} \mathrm{E}^{x} \mathrm{e}^{-r(\tau-t)} f\left(X_{\tau}\right)$ is equivalent to

$$
\sup _{\tau} \mathrm{E}^{x} \int_{t}^{\tau} \mathrm{e}^{-r(s-t)} f\left(X_{s}\right) \mathrm{d} s
$$

and if $\gamma<0$ the problem $\sup _{\tau} \mathrm{E}^{x} \mathrm{e}^{-r(\tau-t)} f\left(X_{\tau}\right)$ is equivalent to

$$
\inf _{\tau} \mathrm{E}^{x} \int_{t}^{\tau} \mathrm{e}^{-r(s-t)} f\left(X_{s}\right) \mathrm{d} s
$$

We conclude the solution for the problems $\sup _{\tau} \mathrm{E}^{x} \int_{t}^{\tau} \mathrm{e}^{-r(s-t)} f\left(X_{s}\right) \mathrm{d} s$ and $\inf _{\tau} \mathrm{E}^{x} \int_{t}^{\tau} \mathrm{e}^{-r(s-t)} f\left(X_{s}\right) \mathrm{d} s$ in the following theorem.

Theorem 14. For a given reward function $f(x)$ in Assumption 1, if $\mathscr{G} f(x)-r f(x)=\gamma f(x)$ and $\gamma>0$, the solution for

$$
w(x)=\sup _{\tau} \mathrm{E}^{x} \int_{t}^{\tau} \mathrm{e}^{-r(s-t)} f\left(X_{s}\right) \mathrm{d} s
$$

has the form

$$
w(x)= \begin{cases}0, & x \leq x^{*}, \\ \frac{f\left(x^{*}\right)}{g\left(x^{*}\right)} \frac{g(x)}{\gamma}-\frac{f(x)}{\gamma}, & x \geq x^{*} .\end{cases}
$$

The optimal stopping time is

$$
\tau^{*}=\inf \left\{s \geq t: X_{s} \leq x^{*}\right\} .
$$

If $\gamma<0$, then the solution for

$$
\widehat{w}(x)=\inf _{\tau} \mathrm{E}^{x} \int_{t}^{\tau} \mathrm{e}^{-r(s-t)} f\left(X_{s}\right) \mathrm{d} s
$$

has the form

$$
w(x)= \begin{cases}0, & x \leq x^{*}, \\ \frac{f\left(x^{*}\right)}{g\left(x^{*}\right)} \frac{g(x)}{\gamma}-\frac{f(x)}{\gamma}, & x \geq x^{*} .\end{cases}
$$

The optimal stopping time is

$$
\tau^{*}=\inf \left\{s \geq t: X_{s} \leq x^{*}\right\} .
$$

The function $g(x)$ is given by

$$
\mathscr{G} g(x)=\operatorname{rg}(x),
$$

satisfying

$$
\begin{aligned}
\lim _{x \rightarrow \infty} g(x) & =0, \\
\frac{\mathrm{d} g(x)}{\mathrm{d} x} & \leq 0, \\
\frac{\mathrm{d}^{2} g(x)}{\mathrm{d} x^{2}} & \geq 0,
\end{aligned}
$$

and the point $x^{*}$ is determined by

$$
\left.\frac{\mathrm{d} f(x)}{\mathrm{d} x}\right|_{x=x^{*}}=\left.\frac{f\left(x^{*}\right)}{g\left(x^{*}\right)} \frac{\mathrm{d} g(x)}{\mathrm{d} x}\right|_{x=x^{*}}, \quad f\left(x^{*}\right)>0 .
$$

Since the expression of $\mathrm{E}^{x} \int_{t}^{\tau} \mathrm{e}^{-r(s-t)} f\left(X_{s}\right) \mathrm{d} s$ can be represented as valuation of equity (see A.3 in Duffie [12]), we will consider the practical application in the stock market in a future work.

\section{Conclusions}

In this paper, we have studied the optimal stopping problems for a continuous-time Markov process, and we have presented the explicit value function and optimal rule.

Three main contributions of this paper are as follows. First, we constructed the value functions and optimal rules for the problems with a large class of reward functions, which are different from the previous researches using some specific functions. With simple conditions for the reward functions, we gave a rigorous mathematical proof to deduce the optimal 
rule based on variational inequalities. Second, we derived the solution of the problem for a general Markov process. Meanwhile, we showed the explicit value functions and optimal stopping times for some concrete Markov processes including diffusion and Lévy processes with jumps. Finally, we linked our results to some optimal problems. Also, we extended the explicit solution of the new optimal problem to a broad class of reward functions and to a general continuoustime Markov process.

\section{Conflicts of Interest}

The author declares that there are no conflicts of interest regarding the publication of this paper.

\section{References}

[1] S. T. Wong, "The generalized perpetual American exchangeoption problem," Advances in Applied Probability, vol. 40, no. 1, pp. 163-182, 2008.

[2] R. McDonald and D. Siegel, "The value of waiting to invest," Quarterly Journal of Economics, vol. 101, no. 4, pp. 707-727, 1986.

[3] Y. Hu and B. Øksendal, "Optimal time to invest when the price processes are geometric Brownian motions," Finance and Stochastics, vol. 2, no. 3, pp. 295-310, 1998.

[4] K. Nishide and L. C. Rogers, "Optimal time to exchange two baskets," Journal of Applied Probability, vol. 48, no. 1, pp. 21-30, 2011.

[5] J. L. Pedersen and G. Peskir, "Solving non-linear optimal stopping problems by the method of time-change," Stochastic Analysis and Applications, vol. 18, no. 5, pp. 811-835, 2000.

[6] I. I. Gihman and A. V. Skorohod, Stochastic Differential Equations. The Theory of Stochastic Processes III, Springer-Verlag, New York, NY, USA, 1979.

[7] S. Christensen and A. Irle, "A harmonic function technique for the optimal stopping of diffusions," Stochastics, vol. 83, no. 4-6, pp. 347-363, 2011.

[8] K. Helmes and R. H. Stockbridge, "Construction of the value function and optimal rules in optimal stopping of onedimensional diffusions," Advances in Applied Probability, vol. 42, no. 1, pp. 158-182, 2010.

[9] T. E. Olsen and G. Stensland, "On optimal timing of investment when cost components are additive and follow geometric diffusions," Journal of Economic Dynamics and Control, vol. 16, no. 1, pp. 39-51, 1992.

[10] S. G. Kou and H. Wang, "First passage times of a jump diffusion process," Advances in Applied Probability, vol. 35, no. 2, pp. 504531, 2003.

[11] N. Cai and S. G. Kou, "Option pricing under a mixedexponential jump diffusion model," Management Science, vol. 57, no. 11, pp. 2067-2081, 2011.

[12] D. Duffie, "Credit risk modeling with affine processes," Journal of Banking and Finance, vol. 29, no. 11, pp. 2751-2802, 2005. 


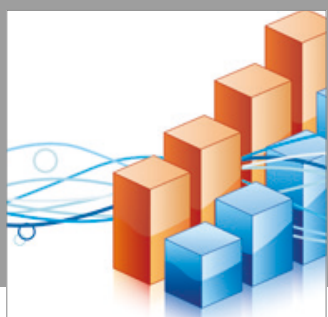

Advances in

Operations Research

vatersals

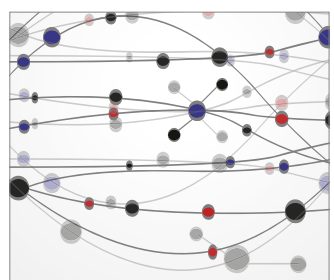

\section{The Scientific} World Journal
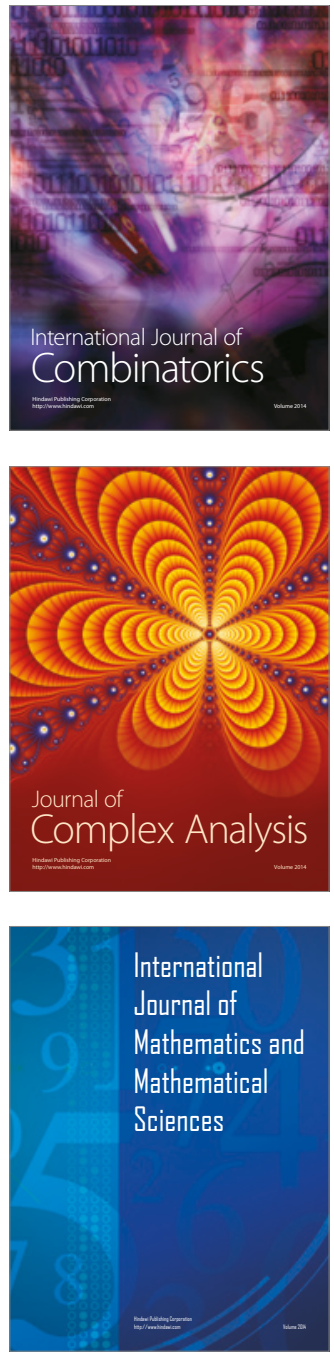
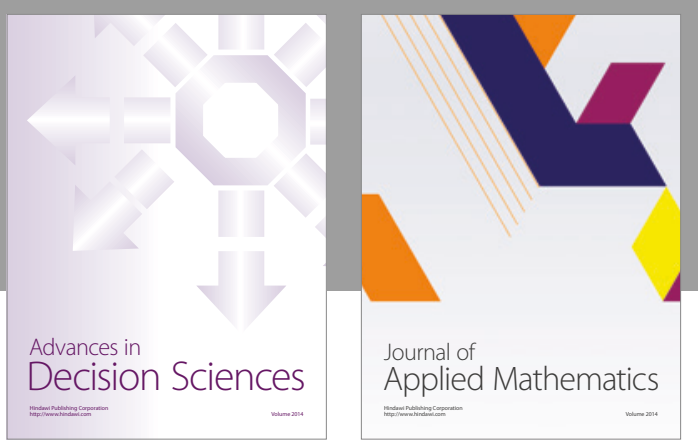

Algebra

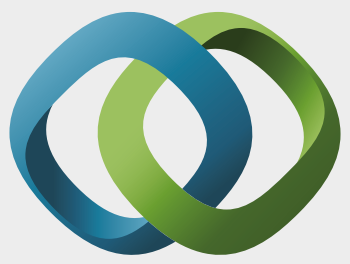

\section{Hindawi}

Submit your manuscripts at

https://www.hindawi.com
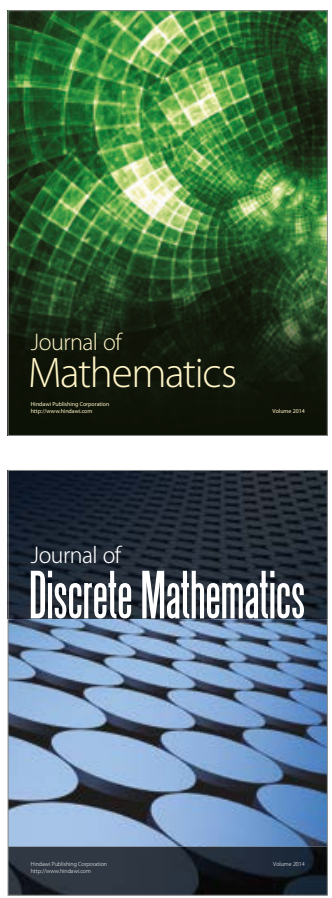

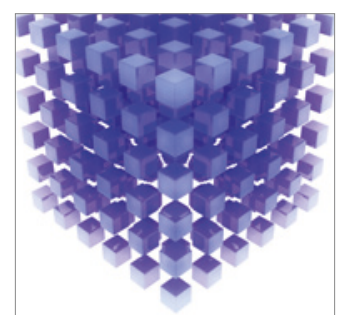

Mathematical Problems in Engineering
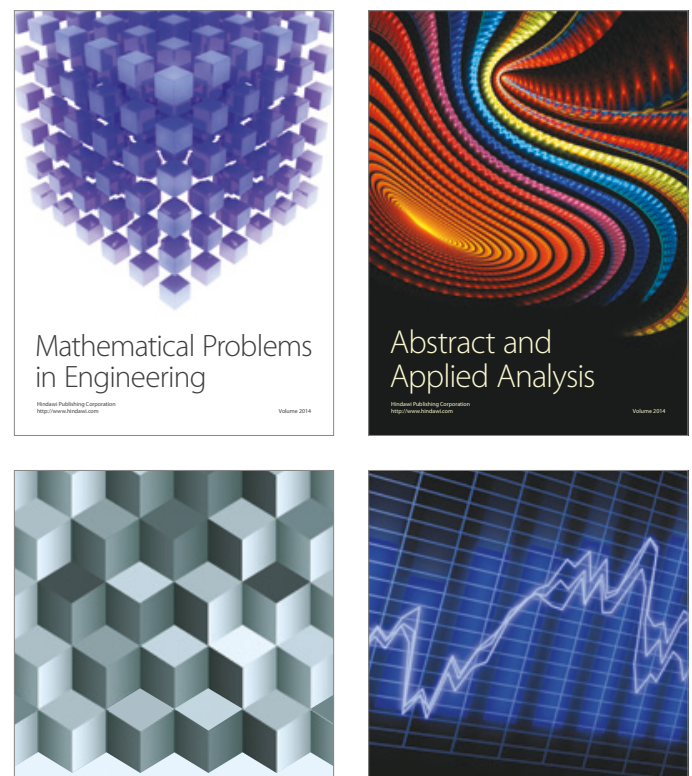

Journal of

Function Spaces

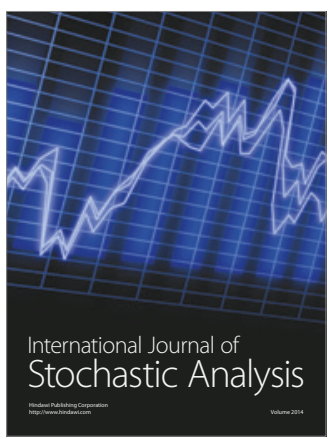

Probability and Statistics
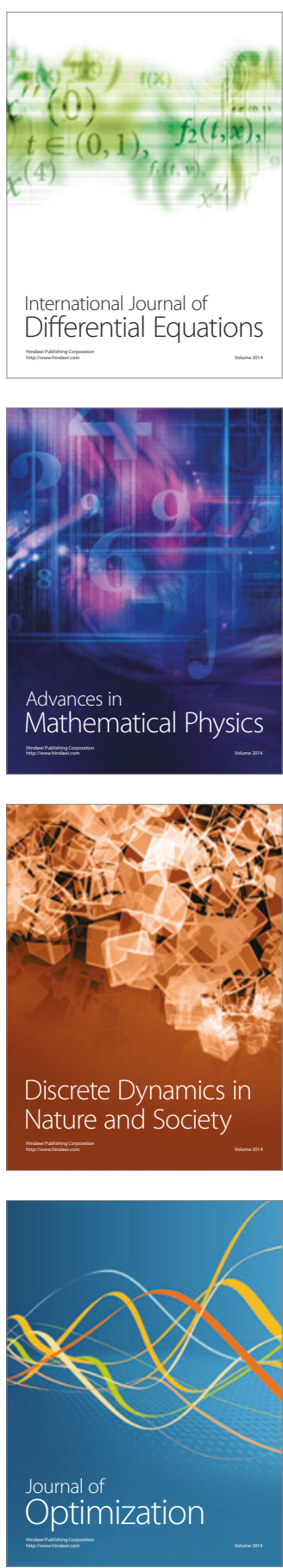